\title{
Network pharmacology analysis reveals neuroprotection of Gynostemma pentaphyllum (Thunb.) Makino in Alzheimer' disease
}

Jiahao Wang ${ }^{1}$, Jiamiao Shi ${ }^{1}$, Ning Jia ${ }^{2^{*}}$ and Qinru Sun ${ }^{3^{*}}$

\begin{abstract}
Background: Alzheimer's disease (AD) is one of the most common neurodegenerative disorders in the world, but still lack of effective drug treatment. Gynostemma Pentaphyllum (Thunb.) Makino (GpM), a Chinese medicinal herb, plays important roles in anti-inflammation, anti-oxidative stress and anti-tumor, which has been reported to ameliorate cognitive impairment of AD. However, the neuroprotective mechanism of GpM remains unclear. This study aims to investigate the targets and possible signaling pathways of GpM in the treatment of AD.

Methods: Active compounds of GpM and their putative target proteins were selected from Traditional Chinese Medicine Systems Pharmacology (TCMSP) Database and Analysis Platform. AD-associated targets were identified from GeneCards, the Online Mendelian Inheritance in Man (OMIM) database and the Therapeutic Target Database (TTD). The intersecting targets of GpM and AD were identified and Gene Ontology (GO), Kyoto Encyclopedia of Genes and Genomes (KEGG) analysis were carried out to analyze the mechanism of them. Compound-target-pathway (CTP) network and protein-protein interaction (PPI) network were constructed and analyzed to elucidate the correlation between compounds, proteins and pathways. Molecular docking was performed to further demonstrate the possibility of GpM for AD.
\end{abstract}

Results: A total of 13 active compounds of $\mathrm{GpM}, 168$ putative target proteins of compounds and 722 AD-associated targets were identified. Eighteen intersecting targets of GpM and AD were found and the epidermal growth factor receptor (EGFR), interleukin-1 beta (IL-1 $\beta$ ), interleukin-6 (IL-6), nitric oxide synthase in endothelial (NOS3) and serum paraoxonase/arylesterase 1 (PON1) were selected as the primary targets of GpM in the treatment of AD. The neuroprotective effect of GPM was related to a variety of pathways, including amoebiasis, HIF-1 signaling pathway, cytokine-cytokine receptor interaction and so on.

Conclusions: Our findings elucidate the active compounds, targets and pathways of GpM involved in effects of antiAD. The novel mechanism of GpM against AD provides more treatment options for AD.

Keywords: AD pathology, Molecular mechanisms, Alzheimer's disease, Gynostemma pentaphyllum (Thunb.) Makino, Network pharmacology

*Correspondence: jianing@mail.xjtu.edu.cn; qinrusun@mail.xjtu.edu.cn 2 Department of Human Anatomy, Histology and Embryology, School of Basic Medical Sciences, Xi'an Jiaotong University Health Science Center, No. 76, West Yanta Road, Xi'an, Shaanxi 710061, People's Republic of China ${ }^{3}$ Institute of Forensic Medicine, Xi'an Jiaotong University Health Science Center, No. 76, West Yanta Road, Xi'an, Shaanxi 710061, People's Republic of China

Full list of author information is available at the end of the article

\section{Background}

Alzheimer's disease (AD) is a neurodegenerative disease with progressive dementia which is characterized by neuronal loss, neuroinflammation and pronounced memory decline. Epidemiological findings show that the incidence rate of AD is increasing with age. Taking 
care of $\mathrm{AD}$ patients require a lot of time and financial resources, which brings huge burden and impact to society and family [1]. Current studies have demonstrated that extracellular accumulation of amyloid beta (A $\beta)$ peptide [2], dysfunction of cholinergic system [3], neurofibrillary tangles (NFTs) of hyper-phosphorylated tau protein [4], and multiple genes including $A B C A 7$, BIN1, CASS4, CD33, CD2AP, CELF1, CLU, CR1 and DSG2 are involved in the occurrence and progression of AD [5]. Substantial studies and clinical trials have demonstrated that it is difficult to develop one medicine for the treatment of $\mathrm{AD}$, because the pathogenesis is very complex, including neurochemicals, amyloid and tau pathological processes, mitochondria, inflammatory pathways and neuroglia [6]. Chinese herbal medicine has a long history and plays a great role in the treatment of nervous system diseases. Its neuroprotective effect in $\mathrm{AD}$ has been confirmed by previous studies [7]. In a recent review, the authors believe that Chinese herbal medicine may be beneficial in improving cognitive function of $\mathrm{AD}$ patients. However, a largescale and multi-center research should be conducted to evaluate its benefits in the treatment of $\mathrm{AD}$ [8]. Therefore, the development of traditional Chinese medicine in the treatment of $\mathrm{AD}$ should be a long-term exploration.

Gynostemma pentaphyllum (Thunb.) Makino (GpM) is one of Chinese medicinal herbs, mainly distributed in northeast and southeast of Asia. Previous studies have shown that GpM has various effects, such as anti-inflammation [9], anti-oxidative stress [10], immune regulation [], anti-cancer [12], anti-aging [13] and prevention of cardiovascular diseases [14]. In recent years, GpM has been shown to alleviate brain lesion in chronic cerebral hypoperfusion [15] and process neuroprotection against 1-methyl-4-phenylpyridinium [16]. It is worth noting that GpM has been shown strong neuroprotective effects in the treatment of AD [1718]. However, the neuroprotective mechanism of GpM in AD has not been fully studied.

In the present study, in order to investigate the potential pharmacological and molecular mechanisms of GpM in the treatment of $\mathrm{AD}$, multiple databases and bioinformatics analysis were applied. The results showed that multiple pathways and targets were involved in the neuroprotection of GpM in the treatment of AD.

\section{Methods}

\section{Screening for active compounds, putative target proteins and AD-associated genes}

The active compounds of GpM and their putative target proteins were obtained from the Traditional Chinese Medicine Systems Pharmacology (TCMSP) Database and Analysis Platform (https://old.tcmsp-e.com/tcmsp.php)
[19]. As a unique database containing a large number of herbs, active ingredients, and their targets, TCMSP contains pharmacokinetic properties of active compounds, such as oral bioavailability (OB), drug-likeness (DL) and so on. Oral bioavailability, as one of the most crucial pharmacokinetic parameters, shows the rate at which drugs enter the blood circulation. The greater the $\mathrm{OB}$ value of a compound, the more likely it is to become an effective drug. The drug-likeness (DL) represents the possibility of the compound becoming a drug. Due to poor pharmacological activity, most compounds in Traditional Chinese Medicine (TCM) cannot become effective drugs. $\mathrm{OB} \geq 30 \%$ and $\mathrm{DL} \geq 0.18$ are considered as the criteria for screening clinical drugs [20]. The putative target proteins of active compounds were collected from TCMSP database, because the database contains the target proteins of each active compound. All information about the active compounds and putative target proteins of GpM was obtained from TCMSP database.

The AD-associated genes in this study were collected from 3 databases, which are GeneCards (http://www.genec ards.org/) [21], Online Mendelian Inheritance in Man (OMIM) (https://omim.org/) [22] database and Therapeutic Target Database (TTD) (http://db.idrblab.net/ttd/) [23].

\section{Gene ontology (GO) analysis and Kyoto encyclopedia of genes and genomes (KEGG) pathway enrichment analysis}

Eighteen overlap targets between 168 putative compound target proteins and $722 \mathrm{AD}$-associated genes were considered as therapeutic targets for $\mathrm{AD}$. In order to elucidate the pathogenesis of $\mathrm{AD}$ and clarify the mechanism and function of GpM, 18 intersecting targets were analyzed by Metascape (http://metascape.org/) [24] Metascape is a website integrating gene annotation and analysis resources with more than 40 databases, combined with GO analysis and KEGG pathway enrichment analysis. Results were considered significant at $P<0.01$.

\section{Compound-target-pathway network establishment}

The information of the components, targets and pathways was intuitively analyzed and shown a compoundtarget-pathway network by using Cytoscape 3.8.2 software (http://www.cytoscape.org/) [25].

\section{Protein-protein interaction (PPI) networks establishment} PPI data were obtained from the STRING database (https://string-db.org/) [26], and the result of PPI data was visualized by Cytoscape software.

\section{Molecule docking}

By using Discovery Studio Client v19.1.0.18287, AutoDockTools-1.5.6 and PyMOL, the binding sites, 


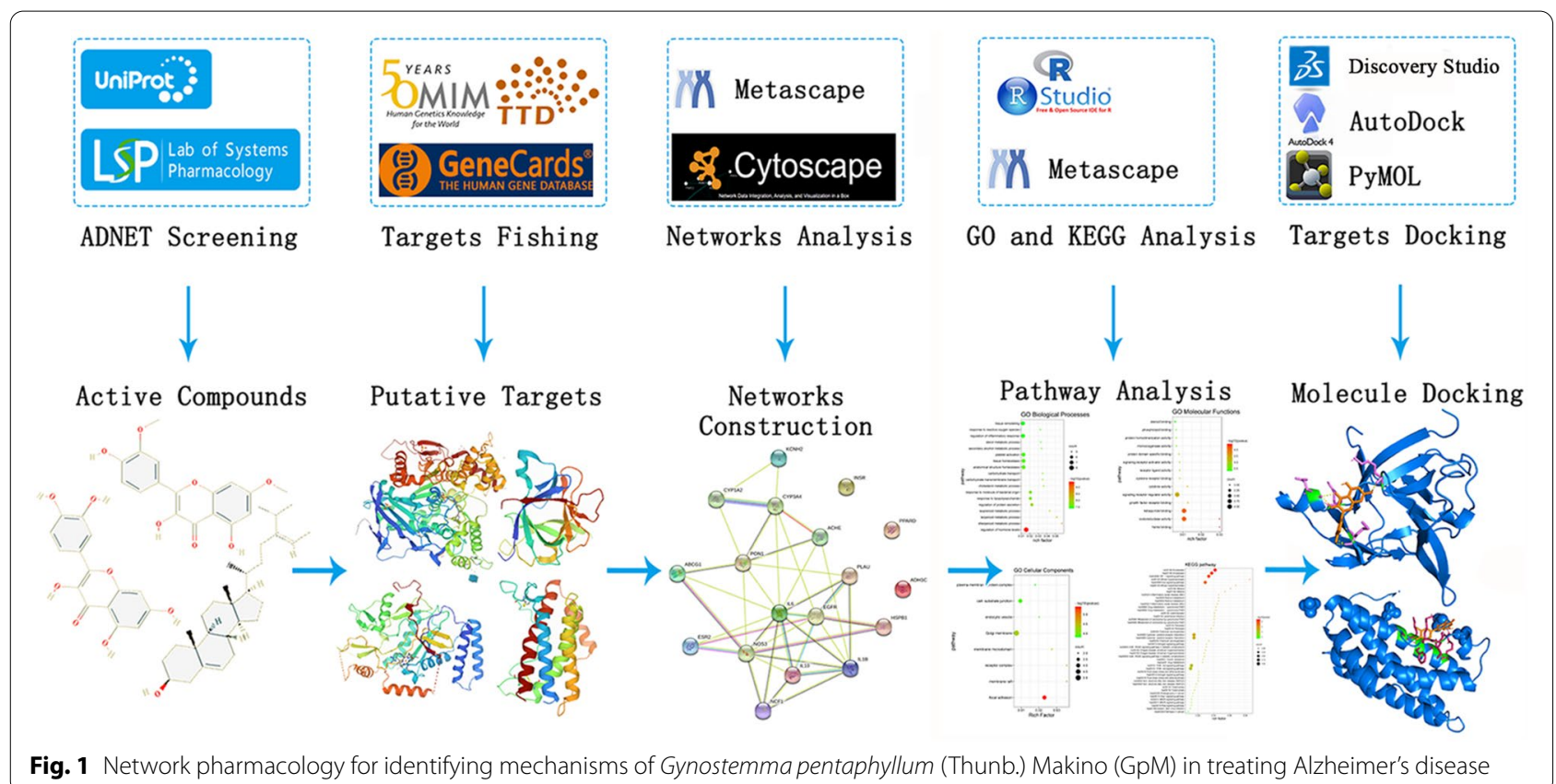

capacities and interactions between compounds and proteins were analyzed [27-29]. The crystal structures of core targets were obtained from the Protein Data Bank (http://www.pdb.org/) [30, 31]. The $3 \mathrm{D}$ chemical structural formulas of quercetin were obtained from PubChem (https://pubchem.ncbi.nlm. nih.gov/) [32].

\section{Results}

\section{Active compounds and putative target proteins}

We used system pharmacology to find potential pharmacological mechanisms, and each step in defining the role of GpM on AD is shown in Fig. 1. Based on the TCMSP database, 13 compounds were selected as active compounds from GpM (Table-S1), as shown in Fig. 2.

The putative target proteins were screened by docking and binding scores. A total of 168 putative target proteins along with the 13 active compounds were captured by using TCMSP (Table-S2). The value of score represents the relationship between the active compound and the target protein. The higher the score is, the closer the relationship is. The 13 active compounds, 168 putative target proteins and their relationships were shown in Fig. 3.

\section{AD-associated targets and the intersecting targets between GpM and AD}

We searched three databases and obtained 722 genes entries as AD-associated targets. Then, 18 overlap targets between 168 putative target proteins of $\mathrm{GpM}$

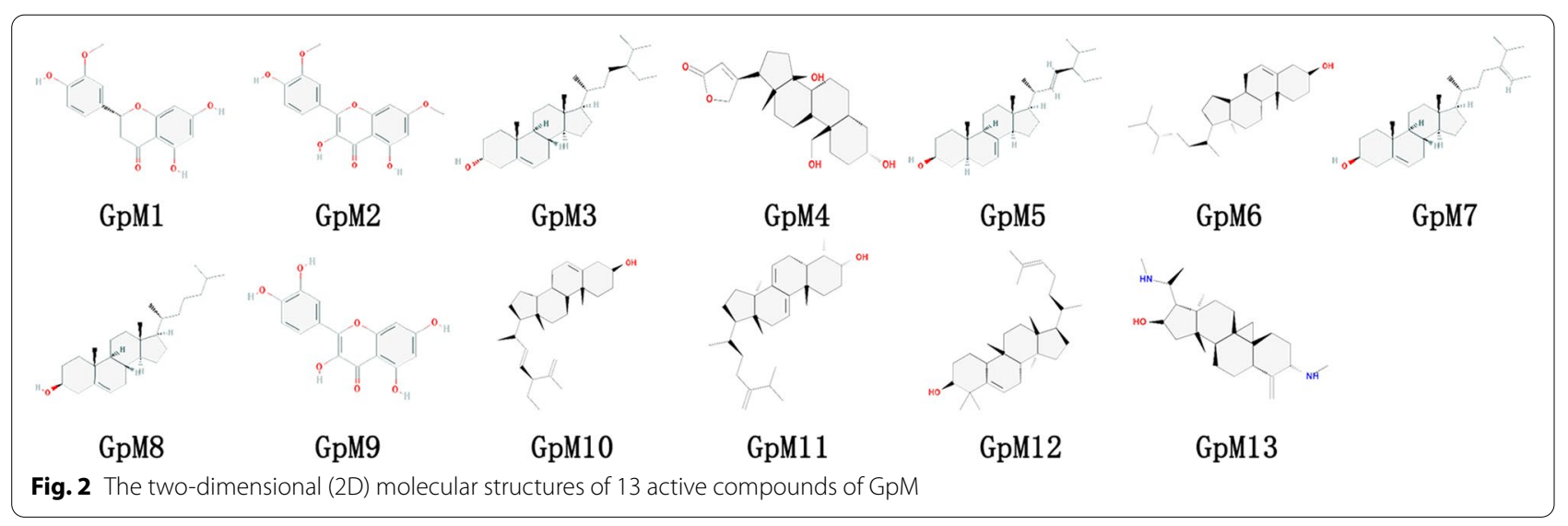




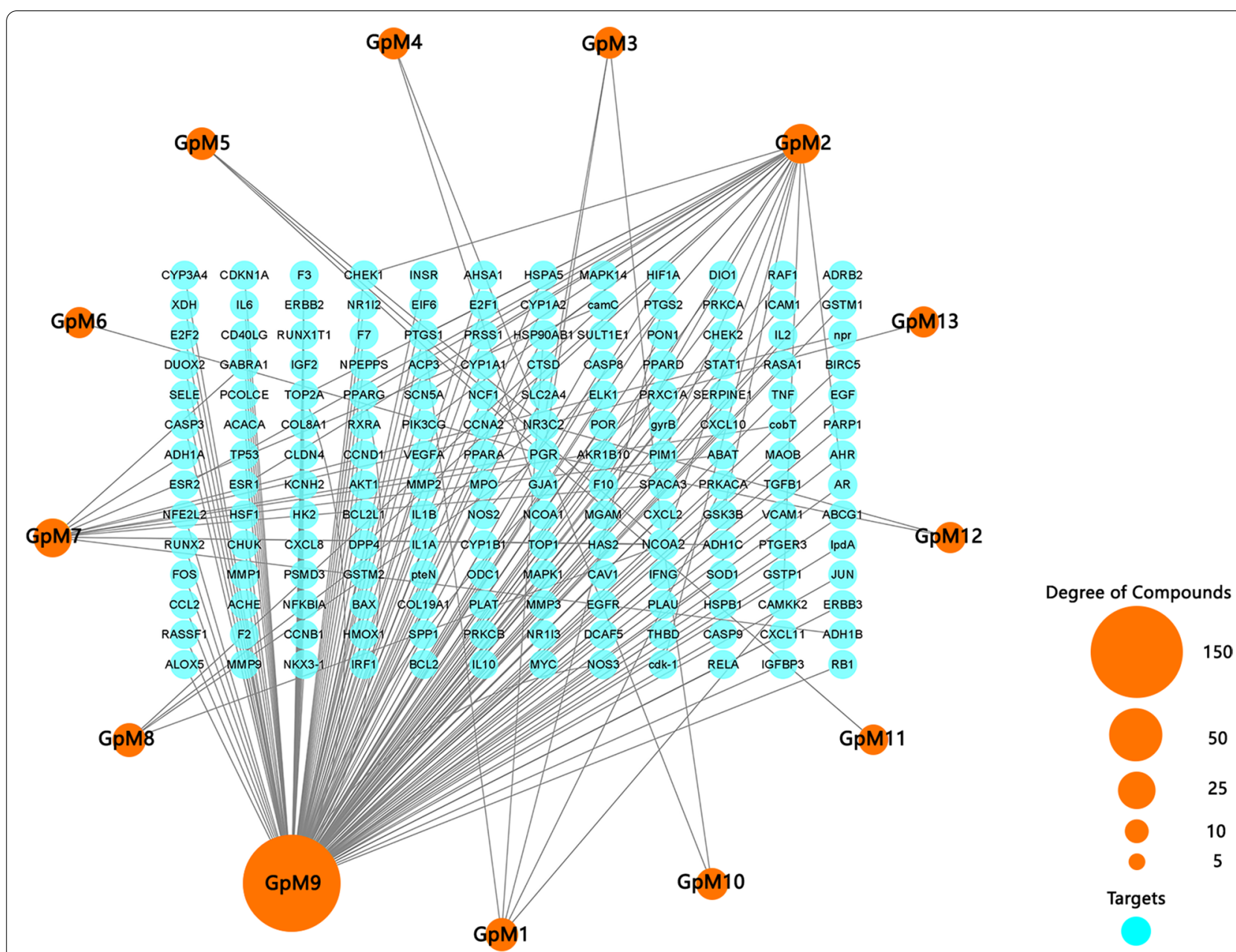

Fig. 3 The construction of Compound-Target network. Different active compounds (the circular nodes with orange) were presented in circle. The size of the circular node represents the targets number of compounds. The putative target proteins (the circular nodes with blue) were listed inside the circle made by active compounds

and $\mathrm{AD}$-associated targets were regarded as intersecting targets (Fig. 4), including ATP-binding cassette sub-family G member 1 (ABCG1), acetylcholinesterase (ACHE), alcohol dehydrogenase $1 \mathrm{C}$ (ADH1C), cytochrome P450 1A2 (CYP1A2), cytochrome P450 3A4 (CYP3A4), epidermal growth factor receptor (EGFR), estrogen receptor beta (ESR2), heat shock protein beta-1 (HSPB1), Interleukin-10 (IL10), interleukin-1 beta (IL-1 $\beta$ ), interleukin-6 (IL-6), Insulin receptor (INSR), potassium voltage-gated channel subfamily $\mathrm{H}$ member 2 (KCNH2), neutrophil cytosol factor 1 (NCF1), nitric oxide synthase, endothelial (NOS3), urokinase-type plasminogen activator (PLAU), serum paraoxonase/arylesterase 1 (PON1), peroxisome proliferator-activated receptor delta (PPARD), listed in Table-S3.

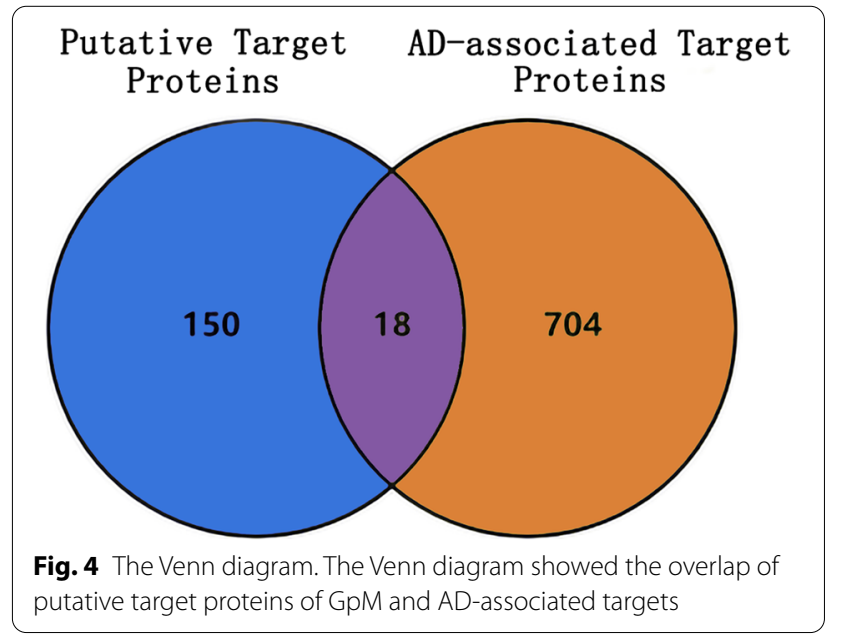


By screening the active components corresponding to the intersecting targets, three compounds, rhamnazin (GpM2), isofucosterol (GpM7) and quercetin (GpM9), were selected to be as the main compounds.

\section{Enrichment analysis of intersecting targets}

GO and KEGG enrichment analyses were performed to identify biological functions and metabolic pathways associated with intersecting targets. Filtering with $P$ value $<0.01$, a minimum count of $3,337 \mathrm{GO}$ entries and 47 KEGG pathway entries were identified (Table-S4, Table-S5). Of the 337 GO entries, there were 314 for biological processes, 8 for cellular components, and 15 for molecular function. Next, we used bubble diagram to visually display the results of GO analysis and KEGG pathway enrichment analysis (Figs. 5, 6, 7 and 8).

As shown in Fig. 5, the results showed that the 15 molecular functions of the intersecting targets of $\mathrm{GpM}$ and $\mathrm{AD}$ mainly concentrated in steroid binding
(GO:0005496), monooxygenase activity (GO:0004497), protein homodimerization activity (GO:0042803), oxidoreductase activity (GO:0016491), heme binding (GO:0020037), growth factor receptor binding (GO:0070851), tetrapyrrole binding (GO:0046906), oxidoreductase activity, acting on paired donors, with incorporation or reduction of molecular oxygen (GO:0016705), signaling receptor regulator activity (GO:0030545), cytokine activity (GO:0005125), cytokine receptor binding (GO:0005126), protein domain specific binding (GO:0019904), phospholipid binding (GO:0005543), receptor ligand activity (GO:0048018) and signaling receptor activator activity (GO:0030546).

As shown in Fig. 6, the 8 cellular components of the intersecting targets of $\mathrm{GpM}$ and $\mathrm{AD}$ were mainly involved in plasma membrane protein complex (GO:0098797), endocytic vesicle (GO:0030139), membrane raft (GO:0045121), membrane microdomain (GO:0098857), focal adhesion (GO:0005925),

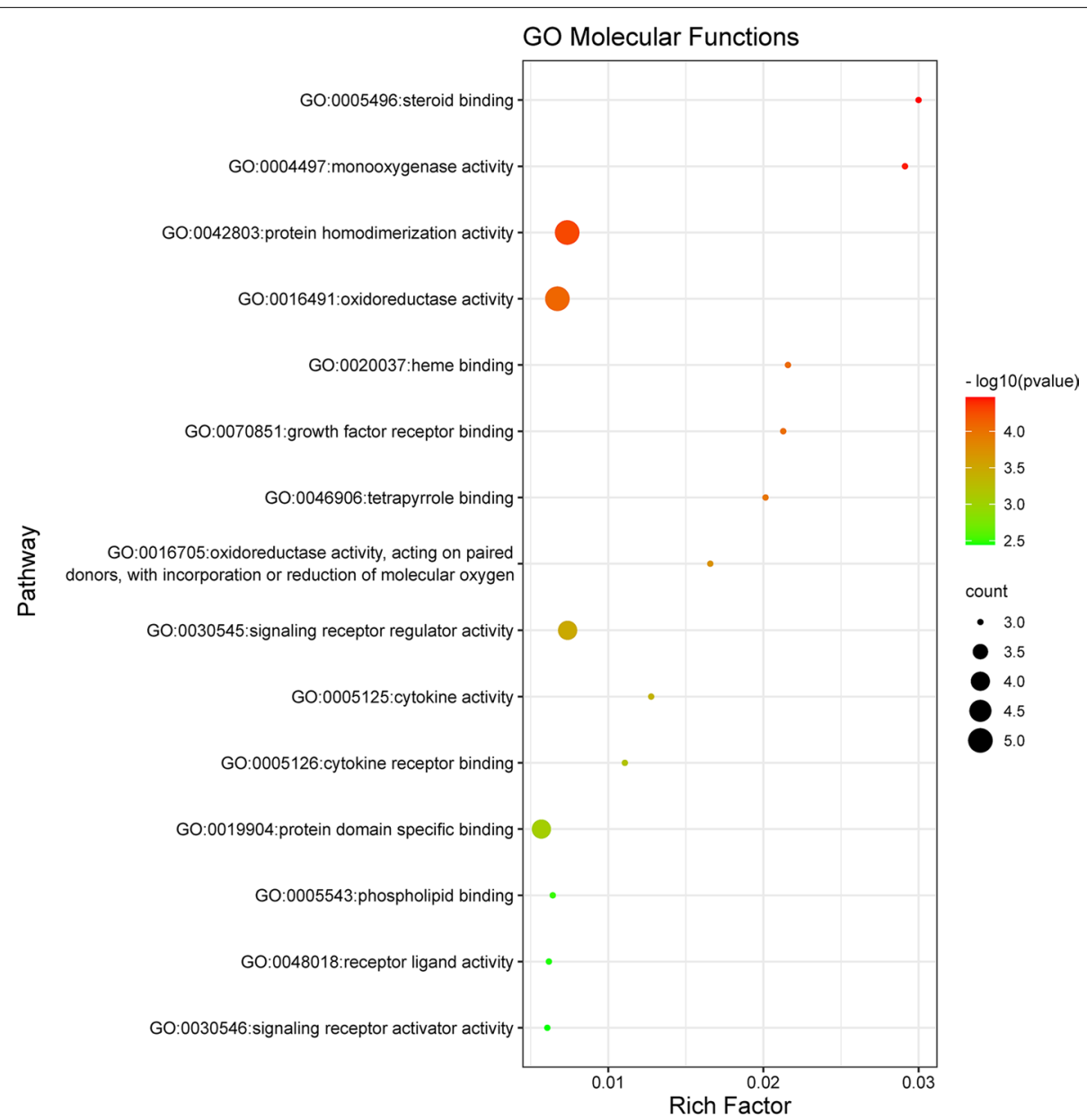

Fig. 5 The molecular functions of Gene Ontology (GO) analysis. The significantly enrichment of molecular functions (MF) with $P<0.01$ 


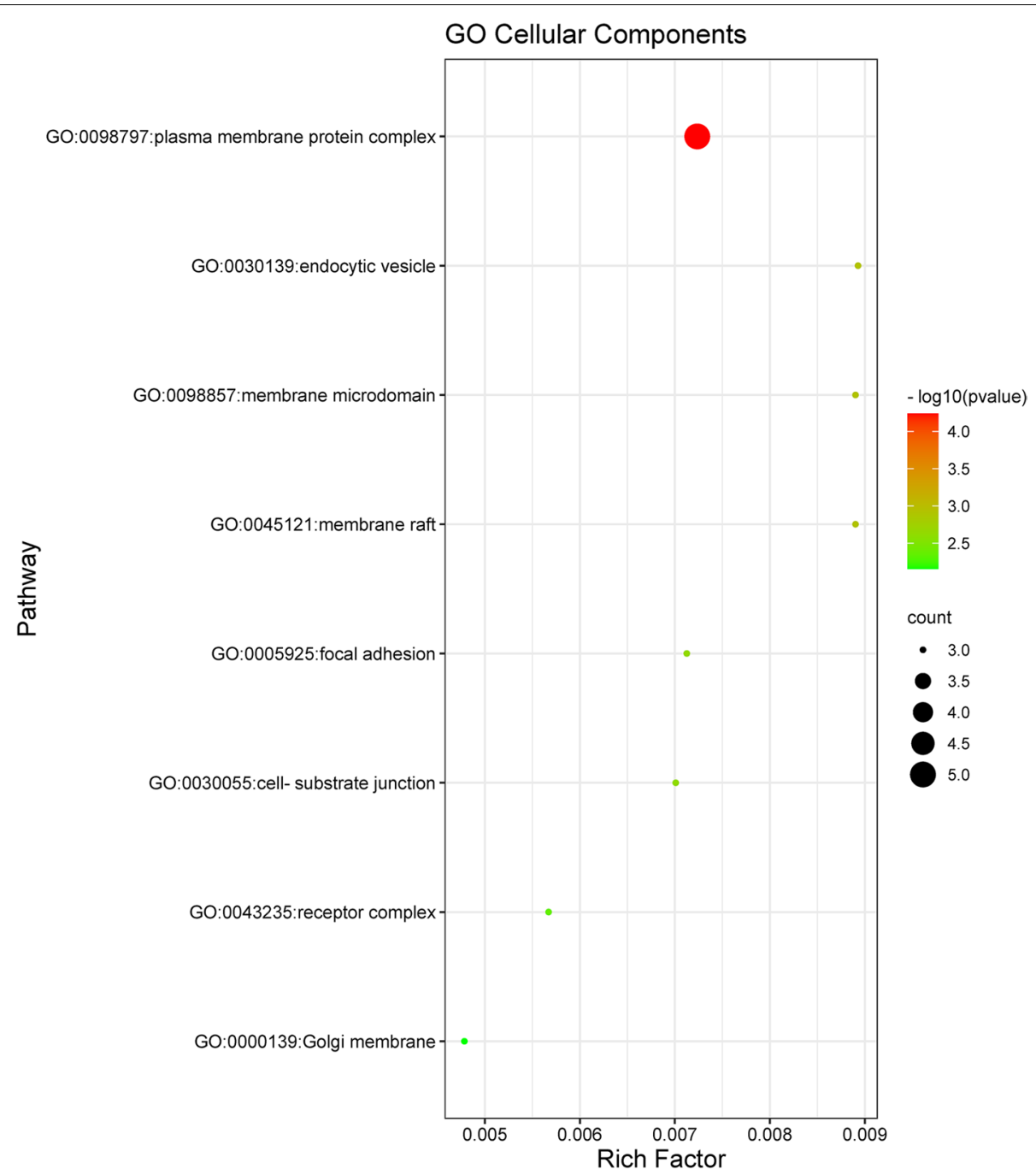

Fig. 6 The cellar components of Gene Ontology (GO) analysis. The significantly enrichment of cellar components (CC) with $P<0.01$

cell-substrate junction (GO:0030055), receptor complex (GO:0043235) and Golgi membrane (GO:0000139).

As represented in Fig. 7, the top 30 biological processes from the intersecting targets of $\mathrm{GpM}$ and $\mathrm{AD}$ were principally linked to regulation of hormone levels (GO:0010817), diterpenoid metabolic process (GO:0016101), terpenoid metabolic process (GO:0006721), isoprenoid metabolic process (GO:0006720), regulation of protein secretion (GO:0050708), cellular response to oxidative stress (GO:0034599), cholesterol metabolic process (GO:0008203), positive regulation of small molecule metabolic process (GO:0062013), response to wounding (GO:0009611), positive regulation of cell migration (GO:0030335), secondary alcohol metabolic process (GO:1902652), sterol metabolic process (GO:0016125), response to inorganic substance (GO:0010035), cellular response to reactive oxygen species (GO:0034614), positive regulation of cell motility (GO:2000147), positive regulation of cellular component movement (GO:0051272), positive regulation of locomotion (GO:0040017), cellular response to chemical stress (GO:0062197), response to lipopolysaccharide (GO:0032496), alcohol metabolic process (GO:0006066), protein secretion (GO:0009306), establishment of protein localization to extracellular region (GO:0035592), response to molecule of bacterial origin (GO:0002237), protein localization to extracellular region (GO:0071692), regulation of lipid localization (GO:1905952), regulation of inflammatory response (GO:0050727), regulation 


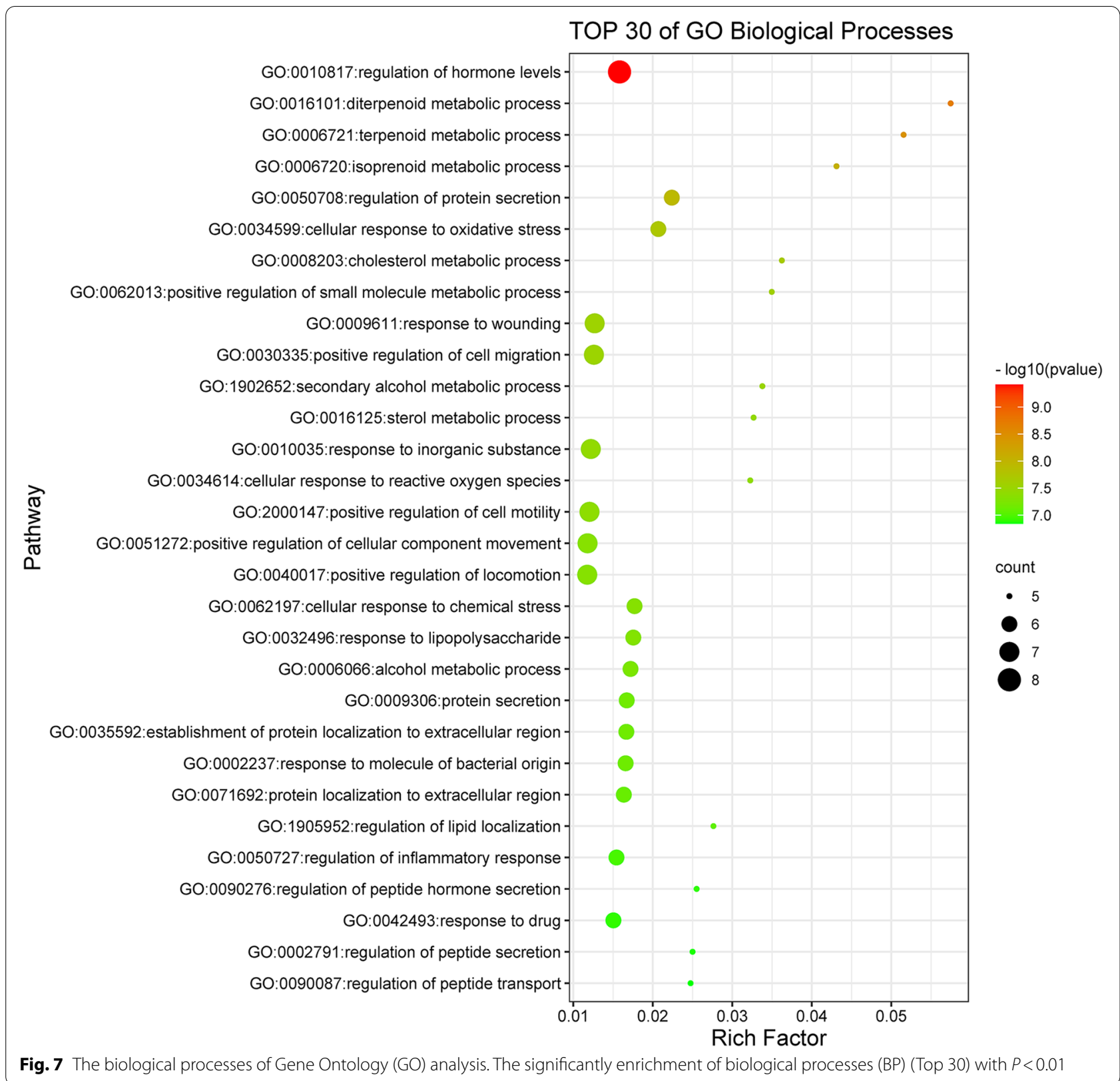

of peptide hormone secretion (GO:0090276), response to drug (GO:0042493), regulation of peptide secretion (GO:0002791) and regulation of peptide transport (GO:0090087).

As shown in Fig. 8, the top 30 KEGG signaling pathways of the intersecting targets were Amoebiasis (ko05146, hsa05146), HIF-1 signaling pathway (hsa04066), foxo signaling pathway (hsa04068), African trypanosomiasis (ko05143, hsa05143), Malaria (ko05144, hsa05144), Inflammatory bowel disease (IBD) (ko05321, hsa05321), Retinol metabolism (hsa00830, ko00830), Drug metabolism - cytochrome P450 (hsa00982, ko00982), Leishmania infection (hsa05140, ko05140), Metabolism of xenobiotics by cytochrome P450 (hsa00980, ko00980), Pertussis (ko05133, hsa05133), Chemical carcinogenesis (ko05204), Cytokine-cytokine receptor interaction (hsa04060, ko04060), Chemical carcinogenesis (hsa05204), Estrogen signaling pathway (ko04915), AGE-RAGE signaling pathway in diabetic complications (ko04933, hsa04933), Chagas disease (American trypanosomiasis) (ko05142, hsa05142) and insulin resistance (hsa04931). 


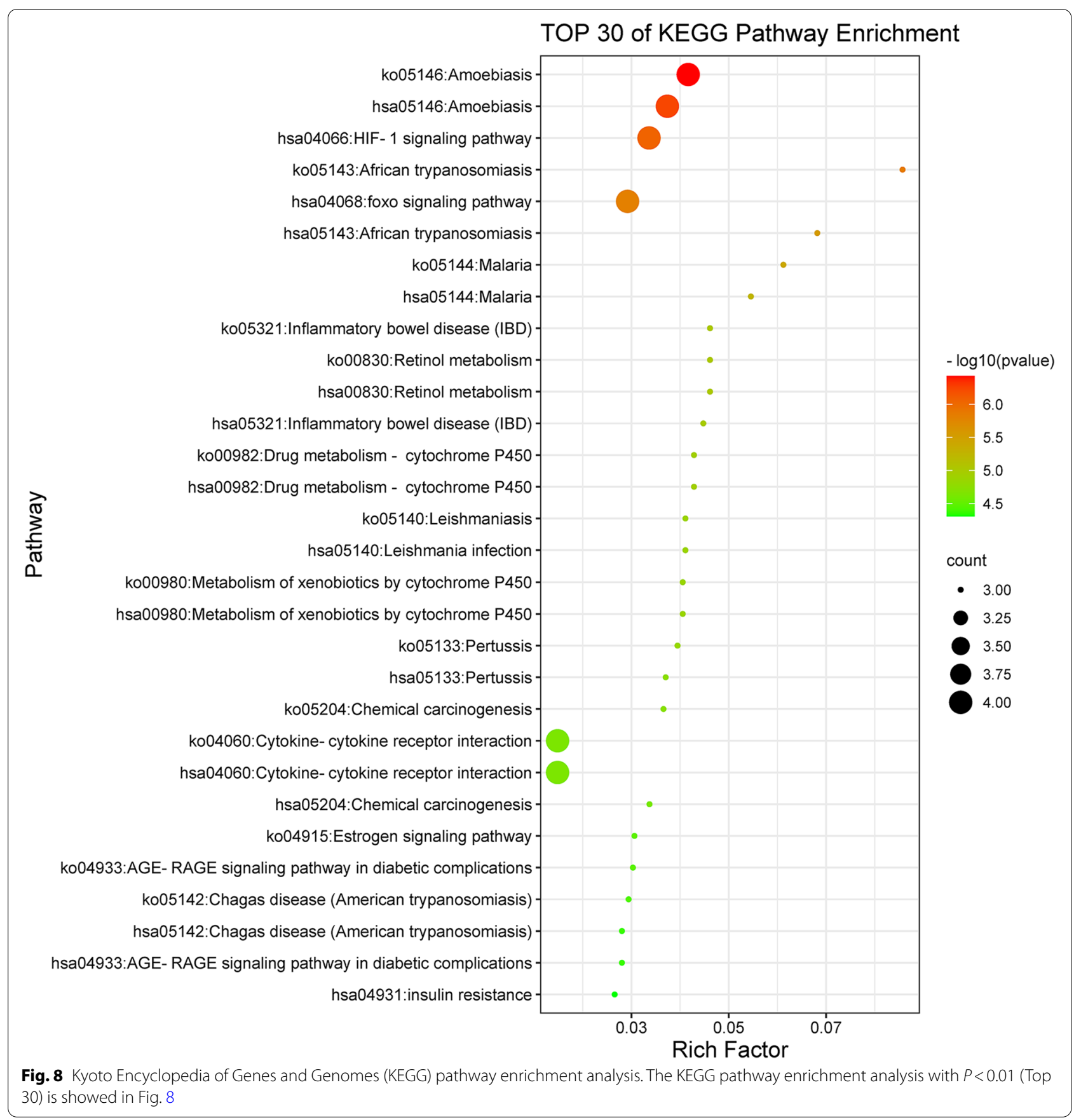

The compound-target-pathway (CTP) and protein-protein interaction (PPI) networks of the intersecting targets

The compound-target-pathway (CTP) and protein-protein interaction (PPI) networks were performed to demonstrate the potential mechanism of GpM on AD. The CTP network showed that 18 proteins and 28 related KEGG pathways (after deleting redundancy) (Fig. 9). The PPI network demonstrated the interaction among the 15 intersecting target proteins (three stray nodes were hidden) (Fig. 10). According to the degree, epidermal growth factor receptor (EGFR), interleukin-1 beta (IL-1ß), interleukin-6 (IL-6), nitric oxide synthase, endothelial (NOS3), serum paraoxonase/arylesterase 1 (PON1) were selected as the core target proteins to determine binding energy by means of a molecular docking analysis. 


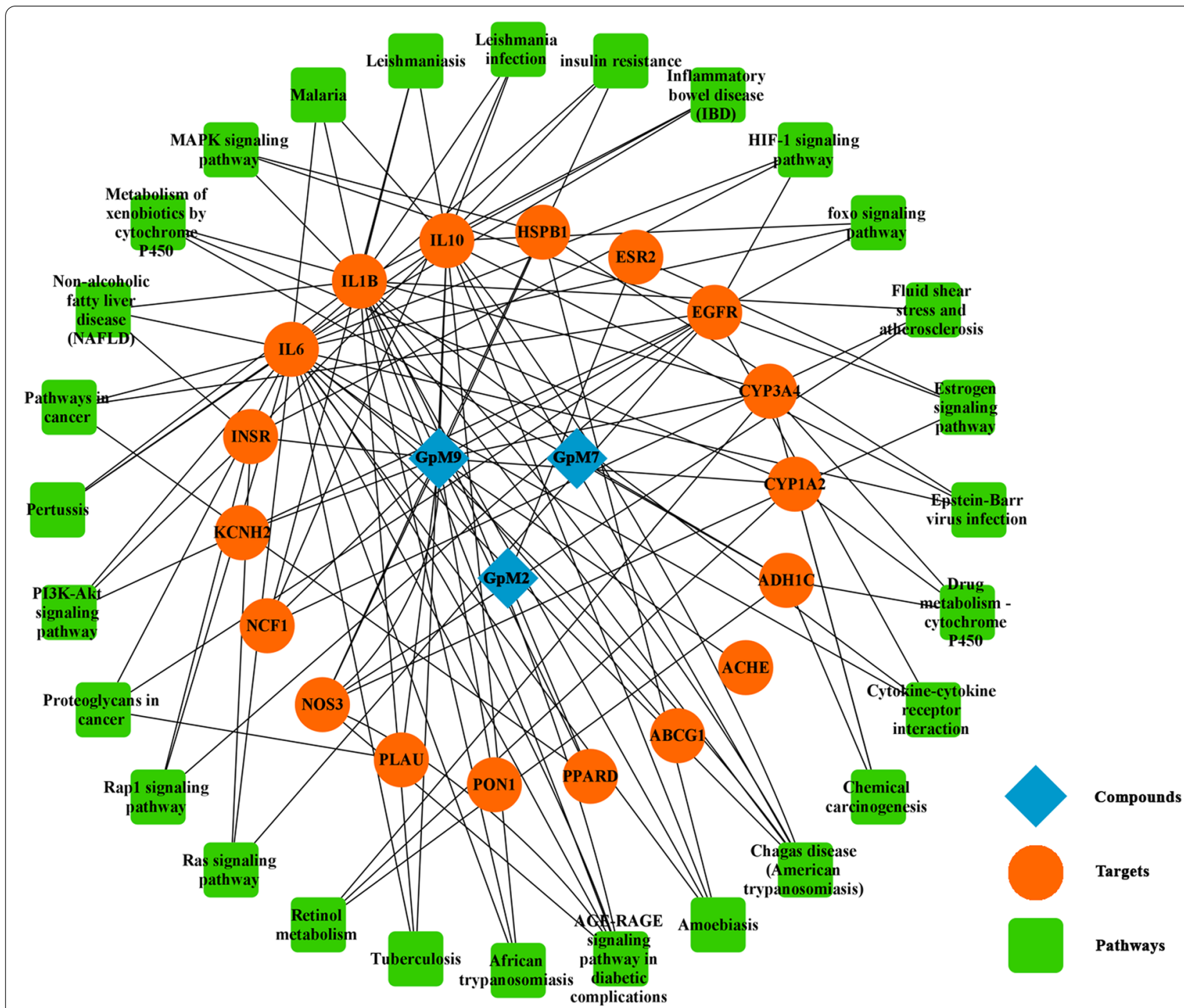

Fig. 9 The compound-target-pathway (CTP) network. The 3 active compounds were presented by blue diamond nodes in the center. The target proteins (orange round nodes) which are associated with AD were situated as a round enclosing the 3 compounds. The pathways were listed at the outermost and presented by green round rectangles

\section{Molecular docking}

To further verifying the function of $\mathrm{GpM}$ on $\mathrm{AD}$, we selected quercetin, one of the three compounds, as the significant compound for the all 5 core targets are part of its putative target proteins. With the utilization of AutoDockTools-1.5.6, OpenBabel-2.4.1 and Pymol-2.2.0 software, the interactions between quercetin and the 5 core targets were analyzed. Figures 11 and 12 showed the molecule docking of 5 core targets with quercetin. The binding energy of each protein with quercetin was shown in Table 1 . The lower binding energy is, the higher the affinity between quercetin and the target (protein) is. The hydrogen bonds were found between the active site of proteins and quercetin.
The 3D model of quercetin in the active site of IL- 6 was showed in the Ray tracing diagram in Fig. 11A. The binding energy of quercetin with IL- 6 was found to be $-7.14 \mathrm{kcal} /$ mol. The type, distance and number of the binding complexes of the active site of IL- 6 and quercetin were shown in the enlarged drawing in Fig. 11B. The following atoms, ARG-179, SER-176, GLU-172, PHE-173, LYS-66 SER-169 and MET-67 formed a pocket around quercetin by hydrogen bonding. Similarly, the binding interaction of quercetin and IL- $1 \beta$ was shown in Fig. 11C. The following atoms, GLU-37, GLN-39, MET-20 and LYS-63 were connected to quercetin by hydrogen bonding (Fig. 11D). The other three docking result were shown in Fig. 12. 


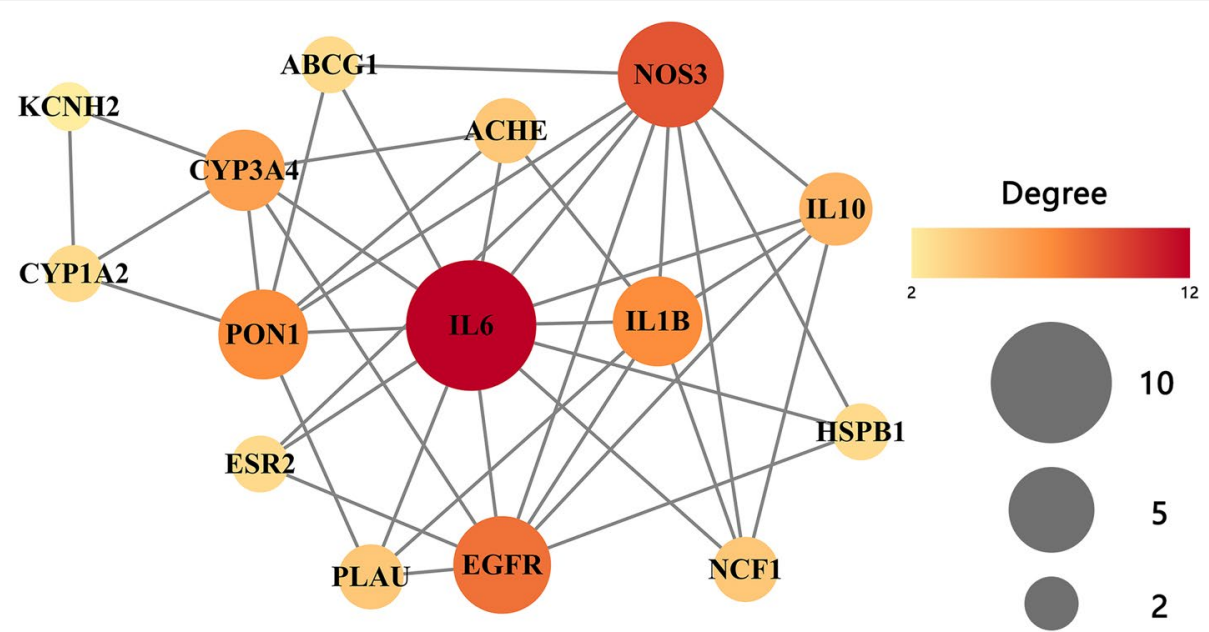

Fig. 10 The Protein-Protein Interaction (PPI) network. The top 15 intersecting target proteins were shown in circular nodes with different gradations of red. The gradation of red and size of the nodes expressed the interaction degree on the network

To further verify the results of molecular docking, we applied Discovery Studio Client to investigate other chemical bonds including Pi-Alkyl, Pi-Sigma and Pi-Pi interaction between quercetin and 5 core targets. The results were shown in Figs. 13 and 14.

Figure 13A showed the 3D model of quercetin and IL-6. To explicitly exhibit the binding environment, 2D model was shown in Fig. 13B. Several bonds were obtained between quercetin and IL- 6 . There were PiAlkyl interactions between quercetin and amino acid residues of IL-6 including LEU-165, LYS-66 and ALA58. Pi-Sigma interactions exited in active sites including GLU-55 and LEU-64. Figure 13C showed the 3D model of quercetin and IL- $1 \beta$. The binding environment between quercetin and IL- $1 \beta$ was shown in Fig. 13D. There were Pi-Alkyl interactions between quercetin and IL-1 $\beta$ at the active sites of LYS-209, PRO-118, ALA-117 and ARG-120. In addition, Pi-Pi interactions existed between quercetin and the active site of HIS-115.

The other three docking results were shown in Fig. 14. All the chemical bonds we found with their bond length were shown in Table 2. Multiple interaction sites and chemical bonds indicated that there was great affinity between quercetin and core targets.

\section{Discussion}

With the development of components analysis of Chinese herbs, TCM shows the characteristics of effective multitarget and multi-component [34]. GpM or some of its components have been shown to possess clinical effects in cardiomyopathy [35], diabetes mellitus [36] and cancer [12]. In recent years, many clinical studies have shown that GpM plays a positive role in alleviating cognitive impairment and brain damage of AD [17, 18]. Although GpM has above medicinal value, the related mechanism remains unclear.

In this study, we explored the active compounds of $\mathrm{GpM}$, and selected the intersecting targets between the compounds and AD. 18 targets from the 168 putative target proteins of compounds and $722 \mathrm{AD}$-associated targets were obtained. Furthermore, multiple signaling pathways, biological processes, cellular components and molecular functions were identified by GO/KEGG pathway enrichment analysis. It is considered that seven pathways were closely related to the pathogenesis of AD including amoebiasis (ko05146, hsa05146), HIF-1 signaling pathway (hsa04066), African trypanosomiasis (ko05143, hsa05143), foxo signaling pathway (hsa04068), malaria (ko05144, hsa05144), inflammatory bowel disease (IBD) (ko05321), retinol metabolism (ko00830, hsa00830). Five essential proteins (IL-6, IL-1 $\beta$, NOS3, PON1 and EGFR) were screened out using CTP and PPI network. Due to good molecular docking results presented by Auto Dock Tools, it is theoretically proved they were target proteins for further study of the anti-AD effects of GpM.

Consistent with our results, HIF-1 signaling pathway and foxo signaling pathway are involved in the occurrence and development of $\mathrm{AD}$, which has been reported in previous studies [37, 38]. Inflammatory bowel disease (IBD) is considered to be closely related 

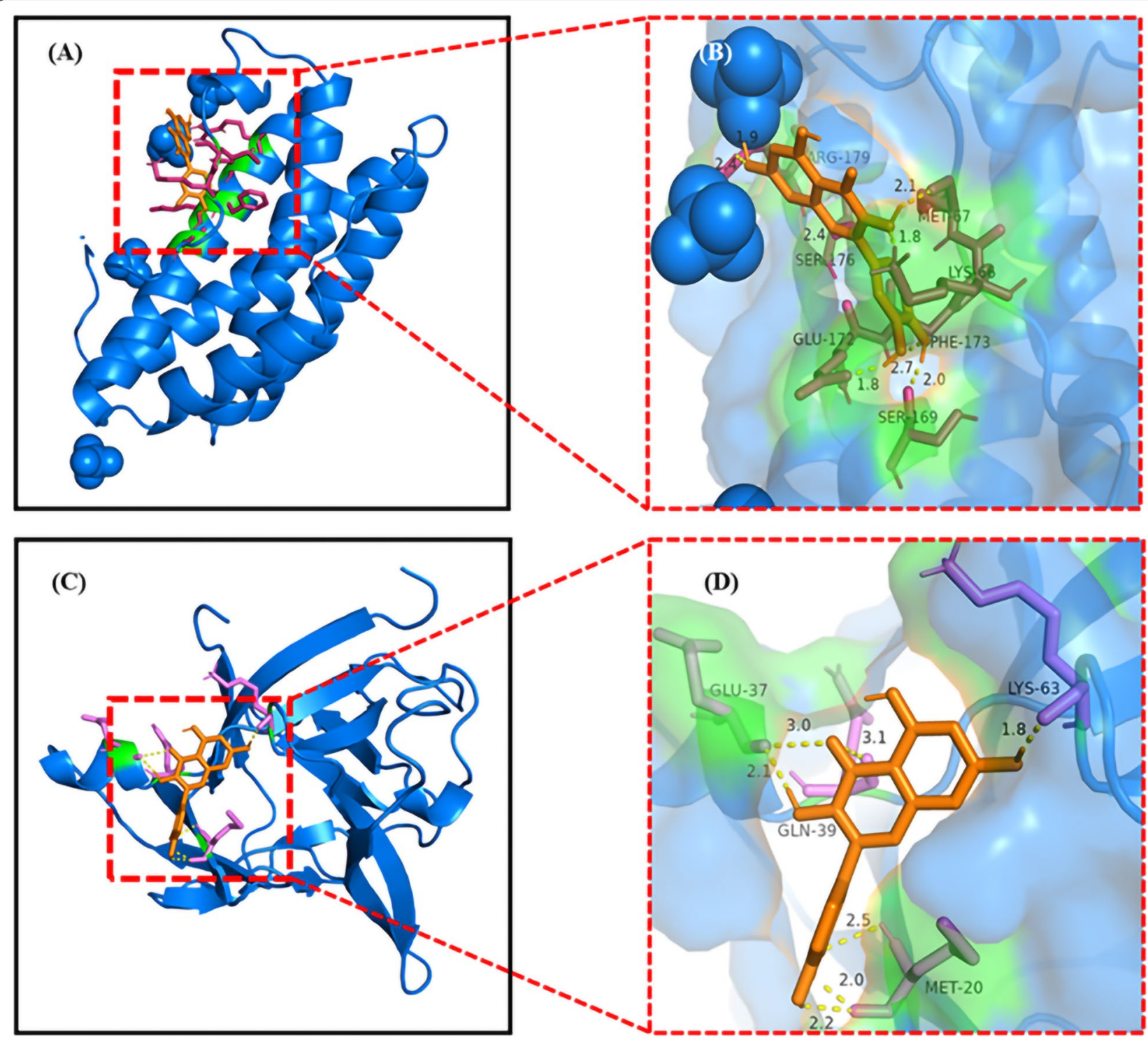

Fig. 11 The molecular docking diagrams of quercetin and IL-6, IL-1 $\beta$ using AutoDockTools. The active sites of the proteins, the binding distances, molecular docking model between the proteins and the quercetin were presented in Fig. 11. (A, B) quercetin and the protein IL-6 (-7.14 kcal/mol); (C), (D) quercetin and the protein $\mathrm{IL}-1 \beta(-6.21 \mathrm{kcal} / \mathrm{mol})$

to pathogenesis of $\mathrm{AD}$, based on studies of gut-brain axis [39]. Additionally, vitamin A deficiency has been demonstrated in patients with AD [40], while vitamin A supplementation can alleviate the development of AD [41]. More interestingly, our study suggests that the $\mathrm{AD}$-associated pathways may relate to amoebiasis, African trypanosomiasis and malaria, which are all caused by parasitic infections and may invade the central nervous system. Existing studies have shown the existence of anti-parasitic infection and anti-AD drugs (such as GSK-3 inhibitors) [42], which imply that the incidence of $\mathrm{AD}$ may relate to parasitic infection. To a certain extent, it shows the credibility and innovation of our conclusion.

Previous studies have shown that inflammatory cytokines are involved in $\mathrm{AD}[43,44]$. A recent study showed that elderly individuals with amyloid-beta deposition had higher levels of IL-1 $\beta$ and IL-6 [45]. In addition, it has been reported that higher level of $A \beta 42$ can reduce endothelial NO synthase (eNOS, NOS3), cyclic GMP 

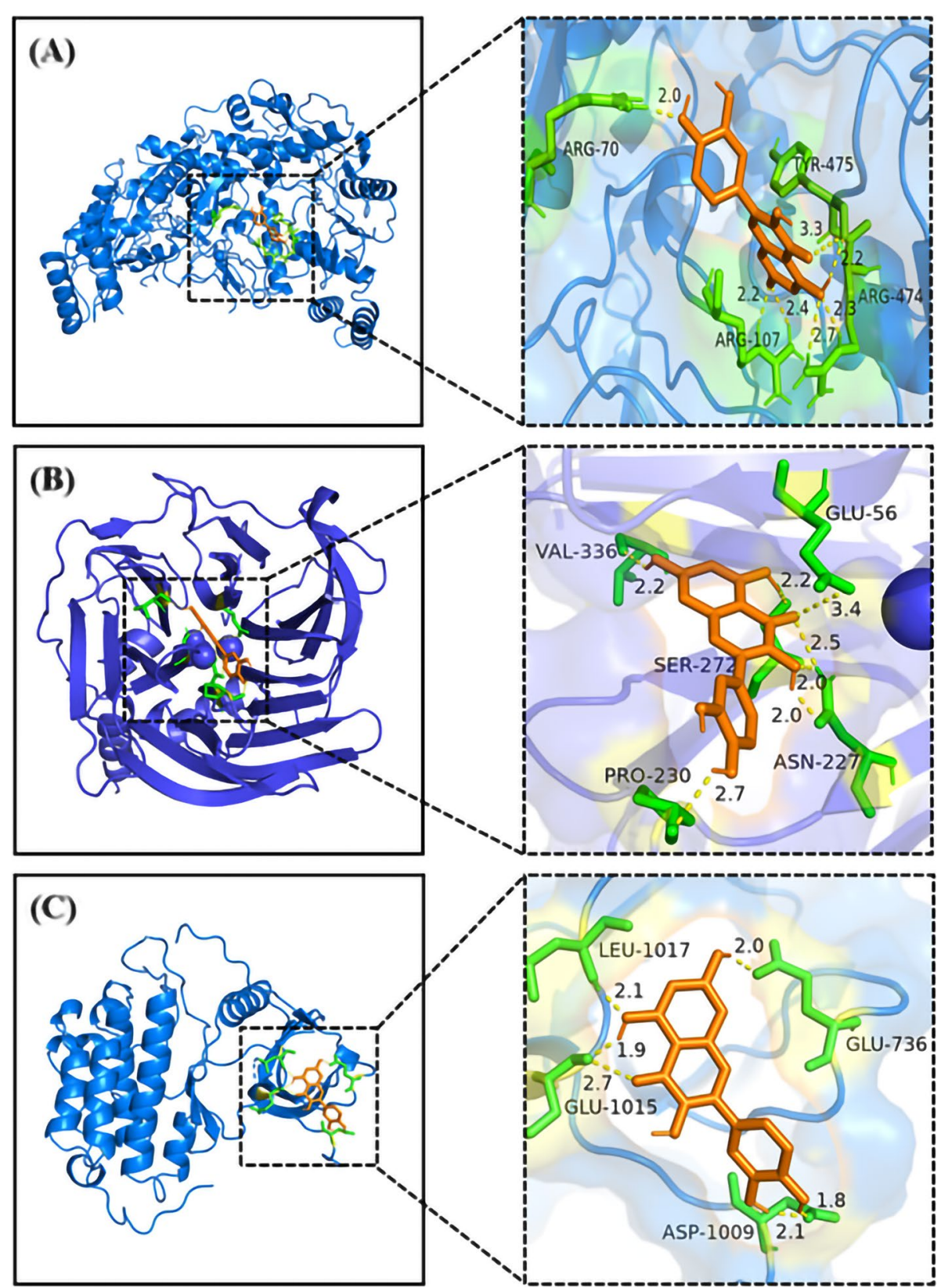

Fig. 12 The molecular docking diagrams of quercetin and NOS3, PON1, EGFR using AutoDockTools. The active sites of the proteins, the binding distances, molecular docking model between the proteins and the quercetin were presented in Fig. 12. (A) quercetin and the protein NOS3 $(-5.59 \mathrm{kcal} / \mathrm{mol}) ;(\mathbf{B})$ quercetin and the protein PON1 $(-6.74 \mathrm{kcal} / \mathrm{mol}) ;(\mathbf{C})$ quercetin and the protein EGFR $(-5.52 \mathrm{kcal} / \mathrm{mol})$

Table 1 The binding energy of quercetin and 5 targets

\begin{tabular}{llllll}
\hline $\begin{array}{l}\text { Binding energy } \\
\text { (kcal/mol) }\end{array}$ & IL-6 & IL-1 $\beta$ & NOS3 & PON1 & EGFR \\
\hline quercetin & -7.14 & -6.21 & -5.59 & -6.74 & -5.52 \\
\hline
\end{tabular}

Note: The binding energy $<-5 \mathrm{kcal} / \mathrm{mol}$ was considered as high affinity between quercetin and the target [33]
(cGMP) and protein kinase G (PKG) activity [46]. This is consistent with our finding that GpM may prevent against $\mathrm{AD}$ by regulating the activity of NOS3 and the levels of IL-1 $\beta$ and IL-6. PON1 is a new factor associated with impaired cognition and may play a role in the development of $\mathrm{AD}$ [47]. It is reported that EGFR is related to AD, and EGFR inhibitors can be used as burgeoning therapeutic 


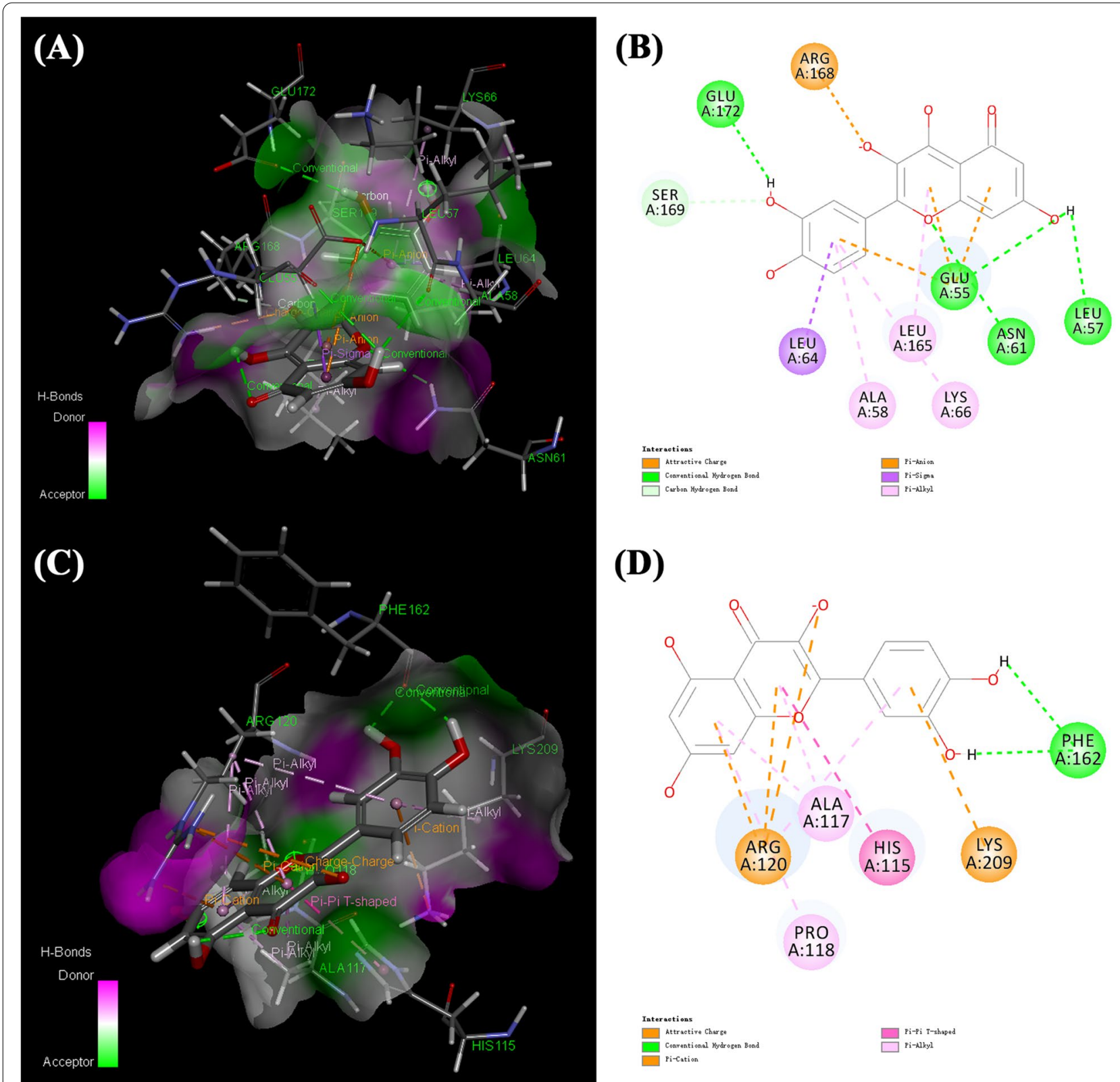

Fig. 13 The molecular docking of quercetin and IL-6, IL-1 $\beta$ using Discovery Studio Client. (A) the 3D model of quercetin and protein IL-6, (B) the 2D model of quercetin and protein IL-6, (C) the 3D model of quercetin and protein IL-1 $\beta,(\mathbf{D})$ the $2 \mathrm{D}$ model of quercetin and protein IL-1 $\beta$

strategy for $\mathrm{AD}$ [48]. These data suggest that targeting on IL-1 $\beta$, IL-6, NOS3, PON1 or EGFR may be effective in the treatment of $\mathrm{AD}$, which supports our finding that proteins with good molecular docking are the important target proteins for neuroprotection of GpM in the treatment of AD.

In conclusion, based on network pharmacology and bioinformatics, this study illustrated the key targets and molecular mechanisms of GpM, which will provide instructive suggestions for the further study of GpM in the treatment of $\mathrm{AD}$.

\section{Conclusions}

In this study, we applied network pharmacology and bioinformatics to analyze the therapeutic effect of $\mathrm{GpM}$ on AD. The active components and putative targets of GpM were explored and discussed systematically. Comparing putative targets of GpM with known AD related genes, constructing and analyzing CTP/PPI networks, 5 important proteins were identified, showing strong therapeutic potentials against AD. Through enrichment analysis, we further identified GpM as a promising drug 


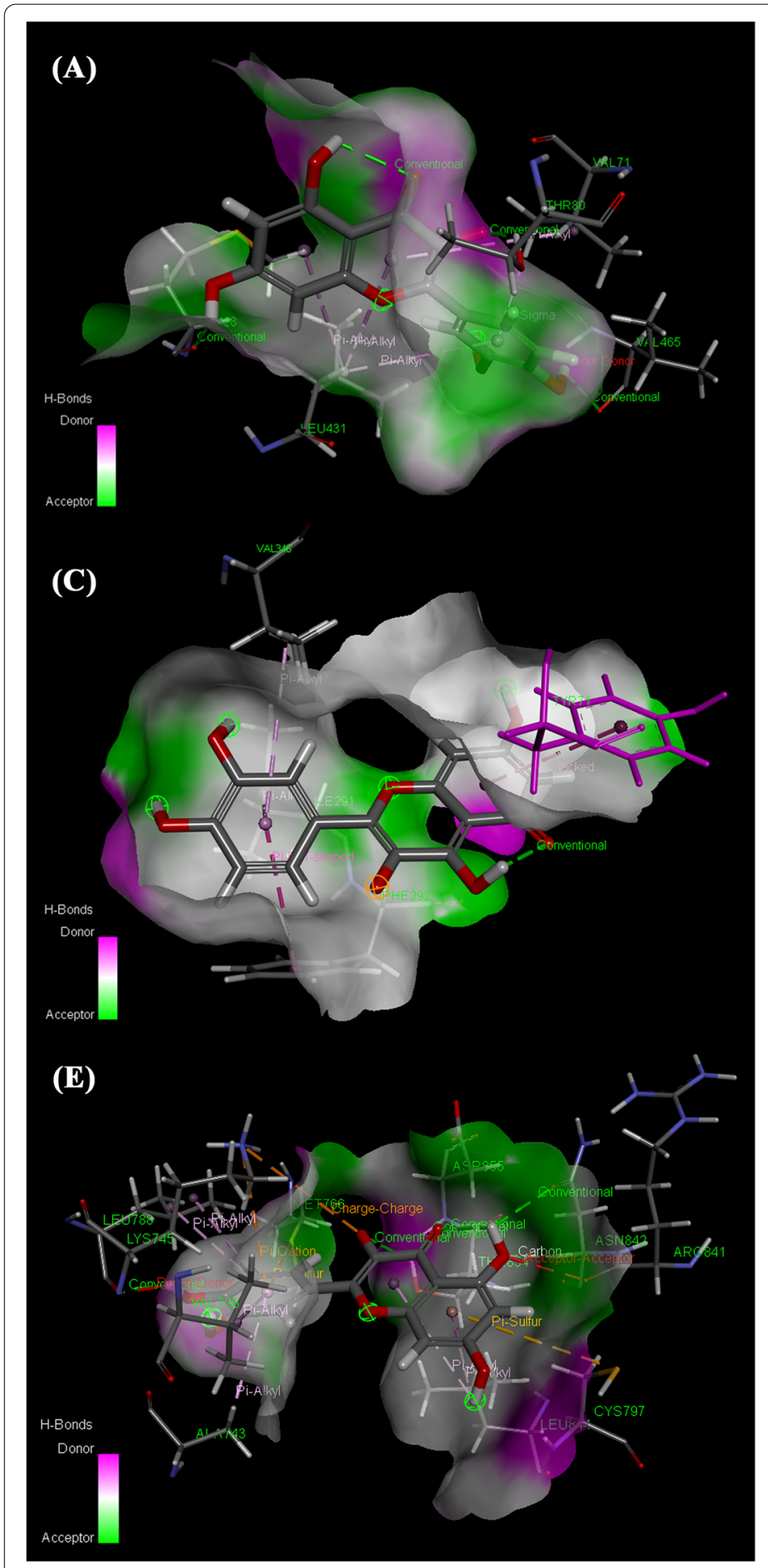

(B)

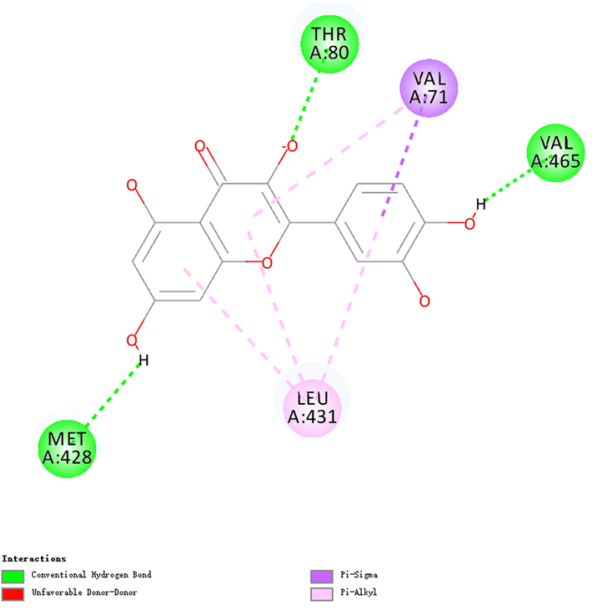

(D)

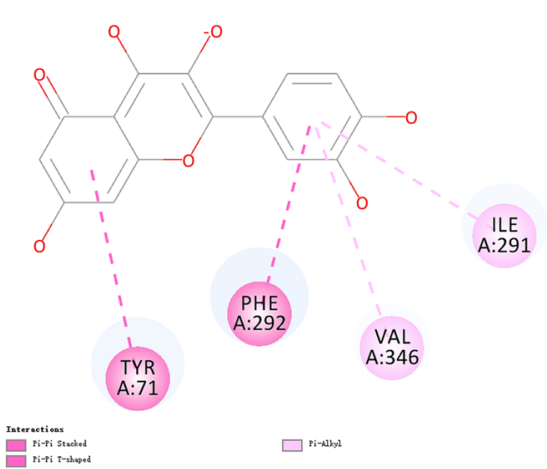

(F)

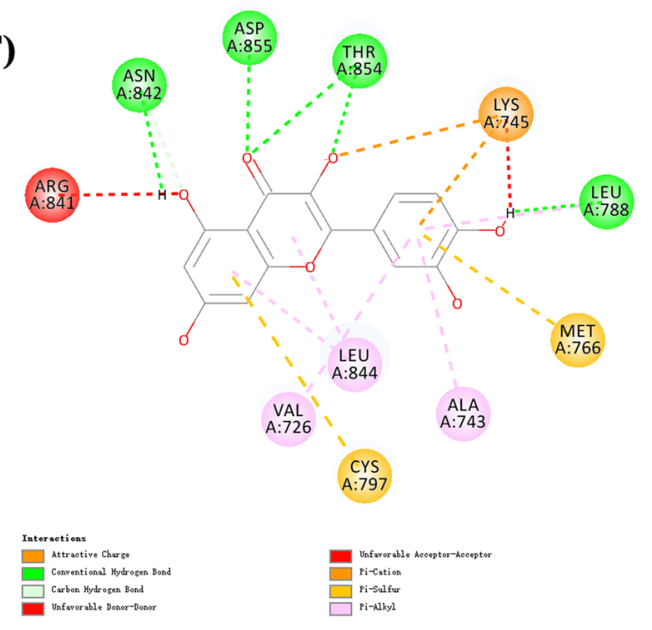

Fig. 14 The molecular docking of quercetin and NOS3, PON1, EGFR using Discovery Studio Client. (A) the 3D model of quercetin and protein NOS3, (B) the 2D model of quercetin and protein NOS3, (C) the 3D model of quercetin and protein PON1, (D) the 2D model of quercetin and protein PON1, (E) the 3D model of quercetin and protein EGFR, (F) the 2D model of quercetin and protein EGFR 
Table 2 Interaction parameters of core targets with quercetin

\begin{tabular}{|c|c|c|c|c|}
\hline Targets & Hydrogen bonding & Pi-Alkyl & Pi-Sigma & $\mathrm{Pi}-\mathrm{Pi}$ \\
\hline $\mathrm{IL}-6$ & $\begin{array}{l}\text { ARG-179(2.4, 1.9) } \\
\text { SER-176(2.4) } \\
\text { GLU-172(1.8) } \\
\text { PHE-173(2.7) } \\
\text { LYS-66(1.8) } \\
\text { MET-67(2.1) } \\
\text { SER-169(2.0) }\end{array}$ & $\begin{array}{l}\text { LEU-165(4.4) } \\
\text { LYS-66(5.3) } \\
\text { ALA-58(4.6) }\end{array}$ & $\begin{array}{l}\text { GLU-55(3.3, 4.1, 4.9) } \\
\text { LEU-64(2.8) }\end{array}$ & - \\
\hline $\mathrm{IL}-1 \mathrm{~B}$ & $\begin{array}{l}\text { GLU-37(3.0, 2.1) } \\
\text { GLN-39(3.1) } \\
\text { MET-20(2.0, 2.2, 2.5) } \\
\text { LYS-63(1.8) }\end{array}$ & $\begin{array}{l}\text { LYS-209(4.9) } \\
\text { PRO-118(5.2) } \\
\text { ALA-117(3.3, 4.0) } \\
\text { ARG-120(3.8, 4.4, 4.8, 5.4) }\end{array}$ & - & HIS-115(4.2) \\
\hline NOS3 & $\begin{array}{l}\text { ARG-70(2.0) } \\
\text { TRY-475(2.2,3.3) } \\
\text { ARG-474(2.3,2.7) } \\
\text { ARG-107(2.2, 2.4) }\end{array}$ & $\begin{array}{l}\text { LEU-431(5.2, 4.9, 4.9) } \\
\text { VAL-71(5.5) }\end{array}$ & VAL-71(2.7) & - \\
\hline PON1 & $\begin{array}{l}\text { GLU-56(3.4) } \\
\text { ASN-227(2.0, 2.0, 2.5) } \\
\text { PRO-230(2.7) } \\
\text { SER-272(2.2) } \\
\text { VAL-336(2.2) }\end{array}$ & $\begin{array}{l}\text { VAL-346(5.2) } \\
\text { ILE-291(4.4) }\end{array}$ & - & $\begin{array}{l}\text { PHE-292(4.5) } \\
\text { TYR-71(4.9) }\end{array}$ \\
\hline EGFR & $\begin{array}{l}\text { LEU-1017(2.1) } \\
\text { GLU-1015(1.9, 2.7) } \\
\text { ASP-1009(1.8, 2.1) } \\
\text { GLU-736(2.0) }\end{array}$ & $\begin{array}{l}\text { LEU-844(4.2, 4.9) } \\
\text { ALA-743(4.7) } \\
\text { VAL-726(5.3) } \\
\text { LYS-745(5.0,4.9) } \\
\text { LEU-788(5.3) }\end{array}$ & - & - \\
\hline
\end{tabular}

with multiple components, targets and pathways for the treatment of AD. In addition, we demonstrated the feasibility of GpM in the treatment of AD by molecular docking. In conclusion, our findings provide a new idea for the neuroprotection of GpM and contribute to the development of GpM in the treatment of AD. However, data mining and analysis alone is not enough. In future, we will conduct experiments to confirm our points and provide more effective treatment measures for $\mathrm{AD}$ patients.

\section{Abbreviations}

AD: Alzheimer's disease; GpM: Gynostemma Pentaphyllum (Thunb.) Makino; GO: Gene Ontology; KEGG: Kyoto Encyclopedia of Genes and Genomes pathway enrichments; CTP: compound-target-pathway; PPI: protein-protein interaction; HIF-1: hypoxia inducible factor-1; EGFR: epidermal growth factor receptor; IL-1 $\beta$ : interleukin-1 beta; IL-6: interleukin-6; NOS3: nitric oxide synthase in endothelial; PON1: serum paraoxonase/arylesterase 1; $A \beta$ : amyloid beta; NFTs: neurofibrillary tangles; ABCA7: ATP-binding cassette transporter A7; BIN1: Bridging integrator 1; CASS4: Cas scaffolding protein family member 4; CD33: Myeloid cell surface antigen CD33; CD2AP: CD2-associated protein; CELF1: CUGBP Elav-like family member 1; CLU: Clusterin; CR1: Complement receptor type 1; DSG2: Desmoglein-2; TCMSP: Traditional Chinese Medicine Database and Analysis Platform; ABCG1: ATP-binding cassette sub-family G member 1; ACHE: acetylcholinesterase; ADH1C: alcohol dehydrogenase 1C; CYP1A2: cytochrome P450 1A2; CYP3A4: cytochrome P450 3A4; ESR2: estrogen receptor beta; HSPB1: heat shock protein beta-1; IL 10: Interleukin-10; INSR: Insulin receptor; $\mathrm{KCNH} 2$ : potassium voltage-gated channel subfamily $\mathrm{H}$ member 2; NCF1: neutrophil cytosol factor 1; PLAU: urokinase-type plasminogen activator; PPARD: peroxisome proliferator-activated receptor delta; TCM: Traditional Chinese Medicine; eNOS: endothelial NO synthase; CGMP: cyclic GMP; PKG: protein kinase G; OB: oral bioavailability; DL: drug-likeness.

\section{Supplementary Information}

The online version contains supplementary material available at https://doi. org/10.1186/s12906-022-03534-z.

Additional file 1: Table-S1. Result of 13 candidate compounds of GpM. Additional file 2: Table-S2. Result of 168 putative target proteins of GpM. Additional file 3: Table-S3. 722 AD-associated target proteins of Homo sapiens from OMIM, TTD and Gene Cards were regarded as the substantially contributing proteins.

Additional file 4: Table-S4: Result of 337 GO terms enrichment of GpM3.

Additional file 5: Table-S5. Result of the KEGG pathway enrichment analysis of GpM.

\section{Acknowledgements}

The authors thank all colleagues at the Xi'an Jiaotong University.

\section{Authors' contributions}

Conceptualization, J.W and J.S; investigation, J.W and J.S; methodology, J.W and J.S; supervision, N.J and Q.S; visualization, J.W and J.S; writing-original draft, J.W and J.S; writing —review and editing, N.J and Q.S. All authors have read and agreed to the published version of the manuscript.

\section{Funding}

This work was supported by the National Natural Science Foundation of China (No.81500927); Key Research and Development Program of Shaanxi (No. 2020SF-133, 2021SF-097); Natural Science Basic Research Plan in Shaanxi Province of China (No.2017JM8017); National Science Foundation for Postdoctoral Scientists of China (No. 2017 M623190, 2018T111074); Foundation for Post-doctoral Scientists of Shaanxi Province (No. 2018BSHERZZ97) and The Fundamental Research Funds for The Central Universities (No. xzy012020084). 


\section{Availability of data and materials}

The datasets used and/or analyzed during the current study available from the corresponding author on reasonable request.

\section{Declarations}

\section{Ethics approval and consent to participate}

Not applicable.

\section{Consent for publication}

Not applicable.

\section{Competing interests}

The authors declare that there are no conflicts of interest.

\begin{abstract}
Author details
${ }^{1}$ Xi'an Jiaotong University Health Science Center , Xi'an, Shaanxi 710061, People's Republic of China. ${ }^{2}$ Department of Human Anatomy, Histology and Embryology, School of Basic Medical Sciences, Xi'an Jiaotong University Health Science Center, No. 76, West Yanta Road, Xi'an, Shaanxi 710061, People's Republic of China. ${ }^{3}$ Institute of Forensic Medicine, Xi'an Jiaotong University Health Science Center, No. 76, West Yanta Road, Xi'an, Shaanxi 710061, People's Republic of China
\end{abstract}

Received: 27 September 2021 Accepted: 14 February 2022

Published online: 07 March 2022

\section{References}

1. 2016 Alzheimer's disease facts and figures. Alzheimer's Dementia 2016, 12(4):459-509.

2. Hardy J, Selkoe DJ: The amyloid hypothesis of Alzheimer's disease: progress and problems on the road to therapeutics. Science (New York, NY) 2002, 297(5580):353-356.

3. Hampel H, Mesulam MM, Cuello AC, Farlow MR, Giacobini E, Grossberg GT, et al. The cholinergic system in the pathophysiology and treatment of Alzheimer's disease. Brain. 2018;141(7):1917-33.

4. Graham WV, Bonito-Oliva A, Sakmar TP. Update on Alzheimer's disease therapy and prevention strategies. Annu Rev Med. 2017;68:413-30.

5. Karch CM, Goate AM. Alzheimer's disease risk genes and mechanisms of disease pathogenesis. Biol Psychiatry. 2015;77(1):43-51.

6. Atri A. Current and future treatments in Alzheimer's disease. Semin Neurol. 2019:39(2):227-40

7. Xu J, Wang F, Guo J, Xu C, Cao Y, Fang Z, Wang Q: Pharmacological Mechanisms Underlying the Neuroprotective Effects of Alpinia oxyphylla Miq. on Alzheimer's Disease. Int J Mol Sci. 2020;21(6):2071

8. Liu N, Zhang T, Sun J, Yao J, Ma L, Fu J, et al. An overview of systematic reviews of Chinese herbal medicine for Alzheimer's disease. Front Pharmacol. 2021:12:761661.

9. Wang $X$, Yang $L$, Yang $L$, Xing F, Yang $H$, Qin $L$, et al. Gypenoside IX suppresses p38 MAPK/Akt/NFKB signaling pathway activation and inflammatory responses in astrocytes stimulated by Proinflammatory mediators. Inflammation. 2017:40(6):2137-50.

10. Zhang GL, Deng JP, Wang BH, Zhao ZW, Li J, Gao L, et al. Gypenosides improve cognitive impairment induced by chronic cerebral hypoperfusion in rats by suppressing oxidative stress and astrocytic activation. Behav Pharmacol. 2011;22(7):633-44.

11. Shang X, Chao Y, Zhang Y, Lu C, Xu C, Niu W: Immunomodulatory and Antioxidant Effects of Polysaccharides from Gynostemma pentaphyllum Makino in Immunosuppressed Mice. Molecules (Basel, Switzerland). 2016;21(8):1085

12. Liu J, Zhang L, Ren Y, Gao Y, Kang L, Qiao Q. Anticancer and immunoregulatory activity of Gynostemma pentaphyllum polysaccharides in $\mathrm{H} 22$ tumor-bearing mice. Int J Biol Macromol. 2014;69:1-4.

13. Phu HT, Thuan DTB, Nguyen THD, Posadino AM, Eid AH, Pintus G. Herbal medicine for slowing aging and aging-associated conditions: efficacy, mechanisms and safety. Curr Vasc Pharmacol. 2020;18(4):369-93.

14. Shaito A, Thuan DTB, Phu HT, Nguyen THD, Hasan H, Halabi S, et al. Herbal medicine for cardiovascular diseases: efficacy, mechanisms, and safety. Front Pharmacol. 2020;11:422.
15. Zhang G, Zhao Z, Gao L, Deng J, Wang B, Xu D, et al. Gypenoside attenuates white matter lesions induced by chronic cerebral hypoperfusion in rats. Pharmacol Biochem Behav. 2011;99(1):42-51.

16. Deng Q, Yang X. Protective effects of Gynostemma pentaphyllum polysaccharides on PC12 cells impaired by MPP(+). Int J Biol Macromol. 2014;69:171-5.

17. Cai H, Liang Q, Ge G. Gypenoside attenuates $\beta$ amyloid-induced inflammation in N9 microglial cells via SOCS1 signaling. Neural plasticity. 2016:2016:6362707.

18. Meng X, Wang M, Sun G, Ye J, Zhou Y, Dong X, et al. Attenuation of Aß2535-induced parallel autophagic and apoptotic cell death by gypenoside $\mathrm{XVII}$ through the estrogen receptor-dependent activation of Nrf2/ARE pathways. Toxicol Appl Pharmacol. 2014;279(1):63-75.

19. Ru J, Li P, Wang J, Zhou W, Li B, Huang C, et al. TCMSP: a database of systems pharmacology for drug discovery from herbal medicines. J Cheminform. 2014;6:13.

20. Xu J, Wang F, Guo J, Xu C, Cao Y, Fang Z, Wang Q: Pharmacological Mechanisms Underlying the Neuroprotective Effects of Alpinia oxyphylla Miq. on Alzheimer's Disease. Int J Mol Sci 2020, 21(6):2071.

21. Stelzer G, Rosen N, Plaschkes I, Zimmerman S, Twik M, Fishilevich $\mathrm{S}$, et al. The GeneCards suite: from gene data mining to disease genome sequence analyses. Curr Protoc Bioinformatics. 2016;54:1.30.31-31.30.33.

22. Hamosh A, Scott AF, Amberger J, Bocchini C, Valle D, McKusick VA. Online Mendelian inheritance in man (OMIM), a knowledgebase of human genes and genetic disorders. Nucleic Acids Res. 2002;30(1):52-5.

23. Wang Y, Zhang S, Li F, Zhou Y, Zhang Y, Wang Z, et al. Therapeutic target database 2020: enriched resource for facilitating research and early development of targeted therapeutics. Nucleic Acids Res. 2020;48(D1):D1031-d1041.

24. Zhou Y, Zhou B, Pache L, Chang M, Khodabakhshi AH, Tanaseichuk O, et al. Metascape provides a biologist-oriented resource for the analysis of systems-level datasets. Nat Commun. 2019;10(1):1523.

25. Shannon P, Markiel A, Ozier O, Baliga NS, Wang JT, Ramage D, et al. Cytoscape: a software environment for integrated models of biomolecular interaction networks. Genome Res. 2003;13(11):2498-504.

26. Szklarczyk D, Gable AL, Lyon D, Junge A, Wyder S, Huerta-Cepas J, et al. STRING v11: protein-protein association networks with increased coverage, supporting functional discovery in genome-wide experimental datasets. Nucleic Acids Res. 2019:47(D1):D607-d613.

27. Morris GM, Huey R, Lindstrom W, Sanner MF, Belew RK, Goodsell DS, et al. AutoDock4 and AutoDockTools4: automated docking with selective receptor flexibility. J Comput Chem. 2009;30(16):2785-91.

28. DeLano W: Use of PyMOL as a communications tool for molecular science. In: Abstracts of Papers of the American Chemical Society: 2004: AMER CHEMICAL SOC 1155 16TH ST, NW, WASHINGTON, DC 20036 USA; 2004: U313-U314.

29. Biovia DS: Discovery studio modeling environment. In: Release; 2017.

30. Somers W, Stahl M, Seehra JS. 1.9 a crystal structure of interleukin 6: implications for a novel mode of receptor dimerization and signaling. EMBO J. 1997:16(5):989-97.

31. Adamek DH, Guerrero L, Blaber M, Caspar DL. Structural and energetic consequences of mutations in a solvated hydrophobic cavity. J Mol Biol. 2005;346(1):307-18

32. Kim S, Chen J, Cheng T, Gindulyte A, He J, He S, et al. PubChem in 2021: new data content and improved web interfaces. Nucleic Acids Res. 2021:49(D1):D1388-d1395.

33. Shi Y, Chen M, Zhao Z, Pan J, Huang S. Network pharmacology and molecular docking analyses of mechanisms underlying effects of the Cyperi Rhizoma Chuanxiong Rhizoma herb pair on depression. Evid Based Complement Alternat Med. 2021;2021:5704578.

34. Chao J, Dai Y, Verpoorte R, Lam W, Cheng YC, Pao LH, et al. Major achievements of evidence-based traditional Chinese medicine in treating major diseases. Biochem Pharmacol. 2017;139:94-104.

35. Zhang H, Chen X, Zong B, Yuan H, Wang Z, Wei Y, et al. Gypenosides improve diabetic cardiomyopathy by inhibiting ROS-mediated NLRP3 inflammasome activation. J Cell Mol Med. 2018;22(9):4437-48.

36. Wang Z, Wang Z, Huang W, Suo J, Chen X, Ding K, et al. Antioxidant and anti-inflammatory activities of an anti-diabetic polysaccharide extracted from Gynostemma pentaphyllum herb. Int J Biol Macromol. 2020;145:484-91. 
37. Baik SH, Kang S, Lee W, Choi H, Chung S, Kim JI, Mook-Jung I: A Breakdown in Metabolic Reprogramming Causes Microglia Dysfunction in Alzheimer's Disease. Cell Metabolism 2019, 30(3):493-507.e496.

38. Manolopoulos KN, Klotz LO, Korsten P, Bornstein SR, Barthel A. Linking Alzheimer's disease to insulin resistance: the FoxO response to oxidative stress. Mol Psychiatry. 2010;15(11):1046-52.

39. Zhang B, Wang HE, Bai YM, Tsai SJ, Su TP, Chen TJ, et al. Inflammatory bowel disease is associated with higher dementia risk: a nationwide longitudinal study. Gut. 2021;70(1):85-91.

40. Lopes da Silva S, Vellas B, Elemans S, Luchsinger J, Kamphuis P, Yaffe K, Sijben J, Groenendijk M, Stijnen T: Plasma nutrient status of patients with Alzheimer's disease: Systematic review and meta-analysis. Alzheimer's Dementia 2014, 10(4):485-502.

41. Ono K, Yamada M. Vitamin a and Alzheimer's disease. Geriatr Gerontol Int. 2012;12(2):180-8.

42. García I, Fall Y, Gómez G: Using topological indices to predict anti-Alzheimer and anti-parasitic GSK-3 inhibitors by multi-target QSAR in silico screening. Molecules (Basel, Switzerland) 2010, 15(8):5408-5422.

43. Glass CK, Saijo K, Winner B, Marchetto MC, Gage FH. Mechanisms underlying inflammation in neurodegeneration. Cell. 2010;140(6):918-34.

44. Nabil KM, Elhady GM, Morsy H: The Association Between Interleukin 1 Beta Promoter Polymorphisms And Keratoconus Incidence And Severity In An Egyptian Population. Clin Ophthalmol (Auckland, NZ) 2019, 13:2217-2223.

45. Flores-Aguilar L, lulita MF, Orciani C, Tanna N, Yang J, Bennett DA, et al. Cognitive and brain cytokine profile of non-demented individuals with cerebral amyloid-beta deposition. J Neuroinflammation. 2021;18(1):147.

46. Meakin PJ, Coull BM, Tuharska Z, McCaffery C, Akoumianakis I, Antoniades $C$, et al. Elevated circulating amyloid concentrations in obesity and diabetes promote vascular dysfunction. J Clin Invest. 2020;130(8):4104-17.

47. Perła-Kaján J, Włoczkowska O, Zioła-Frankowska A, Frankowski M, Smith $A D$, de Jager $C A$, et al. Paraoxonase $1, B$ vitamins supplementation, and mild cognitive impairment. J Alzheimer's Dis. 2021:81(3):1211-29.

48. Mansour HM, Fawzy HM, El-Khatib AS, Khattab MM. Potential repositioning of anti-cancer EGFR inhibitors in Alzheimer's disease: current perspectives and challenging prospects. Neuroscience. 2021;469:191-6.

\section{Publisher's Note}

Springer Nature remains neutral with regard to jurisdictional claims in published maps and institutional affiliations.

Ready to submit your research? Choose BMC and benefit from:

- fast, convenient online submission

- thorough peer review by experienced researchers in your field

- rapid publication on acceptance

- support for research data, including large and complex data types

- gold Open Access which fosters wider collaboration and increased citations

- maximum visibility for your research: over $100 \mathrm{M}$ website views per year

At BMC, research is always in progress.

Learn more biomedcentral.com/submissions 